\title{
Millisecond Magnetar Birth Connects FRB 121102 to Superluminous Supernovae and Long-duration Gamma-Ray Bursts
}

\section{Citation}

Metzger, Brian D., Edo Berger, and Ben Margalit. 2017. "Millisecond Magnetar Birth Connects FRB 121102 to Superluminous Supernovae and Long-Duration Gamma-Ray Bursts." The Astrophysical Journal 841 (1): 14. https://doi.org/10.3847/1538-4357/aa633d.

\section{Permanent link}

http://nrs.harvard.edu/urn-3:HUL.InstRepos:41397481

\section{Terms of Use}

This article was downloaded from Harvard University's DASH repository, and is made available under the terms and conditions applicable to Other Posted Material, as set forth at http:// nrs.harvard.edu/urn-3:HUL.InstRepos:dash.current.terms-of-use\#LAA

\section{Share Your Story}

The Harvard community has made this article openly available.

Please share how this access benefits you. Submit a story.

Accessibility 


\title{
Millisecond Magnetar Birth Connects FRB 121102 to Superluminous Supernovae and Long-duration Gamma-Ray Bursts
}

\author{
Brian D. Metzger ${ }^{1}$, Edo Berger ${ }^{2}$, and Ben Margalit ${ }^{1}$ \\ ${ }^{1}$ Columbia Astrophysics Laboratory, New York, NY 10027, USA
${ }^{2}$ Harvard-Smithsonian Center for Astrophysics, 60 Garden Street, Cambridge, MA 02138, USA \\ Received 2017 January 13; revised 2017 January 28; accepted 2017 February 24; published 2017 May 16
}

\begin{abstract}
Subarcsecond localization of the repeating fast radio burst FRB 121102 revealed its coincidence with a dwarf host galaxy and a steady ("quiescent") nonthermal radio source. We show that the properties of the host galaxy are consistent with those of long-duration gamma-ray bursts (LGRB) and hydrogen-poor superluminous supernovae (SLSNe-I). Both LGRBs and SLSNe-I were previously hypothesized to be powered by the electromagnetic spindown of newly formed, strongly magnetized neutron stars with millisecond birth rotation periods ("millisecond magnetars"). This motivates considering a scenario whereby the repeated bursts from FRB 121102 originate from a young magnetar remnant embedded within a young hydrogen-poor supernova (SN) remnant. Requirements on the gigahertz free-free optical depth through the expanding SN ejecta (accounting for photoionization by the rotationally powered magnetar nebula), energetic constraints on the bursts, and constraints on the size of the quiescent source all point to an age of less than a few decades. The quiescent radio source can be attributed to synchrotron emission from the shock interaction between the fast outer layer of the supernova ejecta with the surrounding wind of the progenitor star, or the radio source can from deeper within the magnetar wind nebula as outlined in Metzger et al. Alternatively, the radio emission could be an orphan afterglow from an initially off-axis LGRB jet, though this might require the source to be too young. The young age of the source can be tested by searching for a time derivative of the dispersion measure and the predicted fading of the quiescent radio source. We propose future tests of the SLSNe-I/LGRB/FRB connection, such as searches for FRBs from nearby SLSNe-I/ LGRBs on timescales of decades after their explosions.
\end{abstract}

Key words: galaxies: active - gamma-ray burst: general - stars: magnetars - stars: neutron - supernovae: general

\section{Introduction}

Fast radio bursts (FRBs) are pulses of coherent gigahertz radio emission with durations of milliseconds or less and inferred dispersion measures $\mathrm{DM} \sim 500-1200 \mathrm{pc} \mathrm{cm}^{-3}$, much larger than expected for propagation through the Galaxy or its halo (Lorimer et al. 2007; Keane et al. 2012; Thornton et al. 2013; Spitler et al. 2014; Ravi et al. 2015; Champion et al. 2016; Petroff et al. 2016; see Katz 2016 for a review). Until recently, little was known about the origin of FRBs, even whether they were of Galactic or extragalactic origin. This uncertainty resulted largely because the bursts were discovered primarily by single dishes with poor angular resolution. Follow-up searches conducted in the error box of FRB 150418 led to a claimed detection of a host galaxy and a coincident steady radio counterpart (Keane et al. 2016); however, this association was disputed by Williams \& Berger (2016), who showed that the radio counterpart was an active galactic nucleus (AGN) with a significant probability of being located by chance within the FRB sky error region (see also Johnston et al. 2017).

Although most FRBs are detected as single events, it was discovered that several repeat with consistent DM and sky localization (Spitler et al. 2014, 2016; Scholz et al. 2016). Using fast-dump interferometry with the Karl G. Jansky Very Large Array (Law et al. 2015), FRB 121102 was recently localized to $\approx 0.1$ arcsec precision (Chatterjee et al. 2017). Optical imaging and spectroscopy by Tendulkar et al. (2017) identify an extended source coincident with the burst displaying prominent Balmer and [O III] at a redshift of $z=0.19273$, indicating a dwarf galaxy of estimated stellar mass $M_{\star} \approx 4-7 \times 10^{7} M_{\odot}$ at a luminosity distance of $D=972 \mathrm{Mpc} \simeq 3 \times 10^{27} \mathrm{~cm}$. Based on the $\mathrm{H} \alpha$ flux of the galaxy, Tendulkar et al. (2017) infer a total star formation rate of $0.4 M_{\odot} \mathrm{yr}^{-1}$, which they use to estimate a maximum DM through the plane of the galaxy of $324 \mathrm{pc} \mathrm{cm}^{-3}$. This is much higher than the residual (host + local) dispersion measure $\mathrm{DM}_{\text {host }+ \text { local }}=55-225 \mathrm{pc} \mathrm{cm}^{-3}$ (Tendulkar et al. 2017) when contributions from the Galaxy, Galactic halo, and intergalactic medium are subtracted from the measured DM.

In addition to localizing FRB 121102, Chatterjee et al. (2017) identify a coincident continuum radio source with a nearly flat spectrum $F_{\nu} \approx \nu^{\beta}$ in the frequency range $\nu=1.6-22 \mathrm{GHz}$ with $\beta \approx-0.2$ and a $1.7(5 \mathrm{GHz})$ luminosity of $\nu L_{\nu} \approx 3(7) \times 10^{38} \mathrm{erg} \mathrm{s}^{-1}$, though the spectrum appears to steepen at the highest frequencies $\nu \gtrsim 10 \mathrm{GHz}$. Marcote et al. (2017) use VLBI observations to show that the quiescent radio source is colocated with the burst to $<40 \mathrm{pc}$ (Marcote et al. 2017 ) and a projected size constraint at $5 \mathrm{GHz}$ of $\lesssim 0.7 \mathrm{pc}$. Both radio sources are offset by $\sim 0.5-1 \mathrm{kpc}$ from the light centroid of the host galaxy (Tendulkar et al. 2017), potentially disfavoring an AGN or galactic nuclear origin (though the morphology of dwarf galaxies is highly irregular, and hence their nuclei are not easy to locate). Observations by Chandra place an upper limit of $L_{\mathrm{X}} \lesssim 5 \times 10^{41} \mathrm{erg} \mathrm{s}^{-1}$ on the luminosity of spatially coincident $\mathrm{X}$-ray emission, also in tension with attributing the compact radio source to an AGN (Chatterjee et al. 2017).

The repetition and energetics of the bursts from FRB 121102 were used by Marcote et al. (2017) and Tendulkar et al. (2017) to argue for a possible origin associated with a young neutron star or magnetar (Kulkarni et al. 2015; Katz 2016; Cordes \& Wasserman 2016; Lyutikov et al. 2016; Popov \& Pshirkov 2016; Yang et al. 2016), embedded within the ejecta shell of a young supernova (SN) remnant (Connor et al. 2016; Piro 2016; 

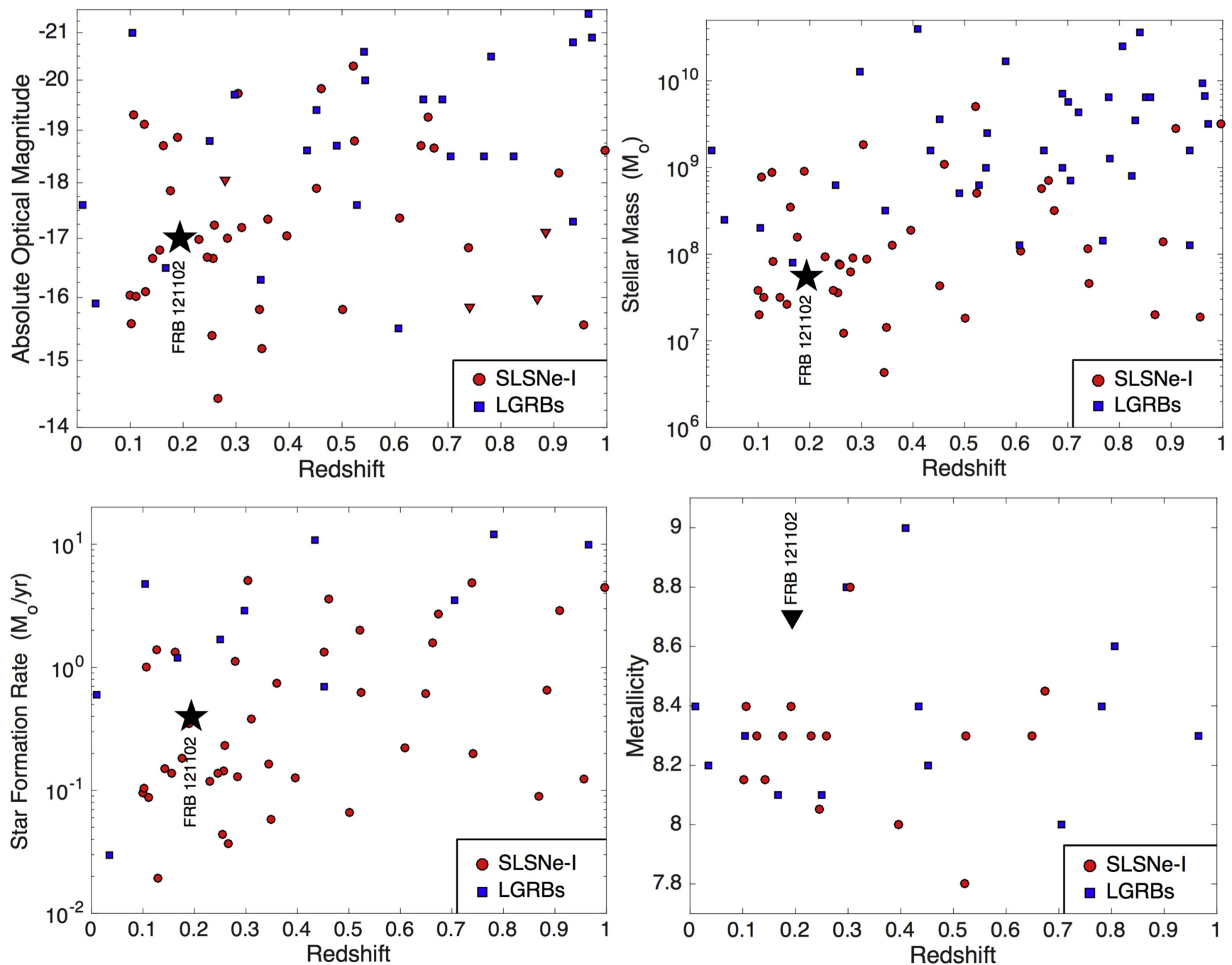

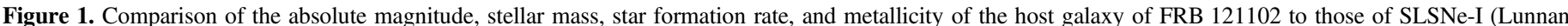

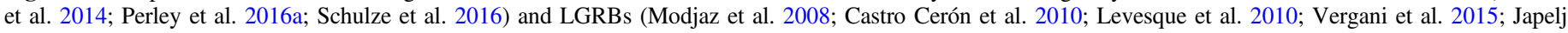
et al. 2016; Perley et al. 2016b).

Murase et al. 2016). In this paper we present further evidence in favor of an association between FRB 121102 and the birth of a young magnetar. In Section 2 we show quantitatively that the host galaxy of FRB 121102 is consistent with that of hydrogenpoor superluminous SNe (SLSNe-I; Quimby et al. 2011; GalYam 2012) and long-duration gamma-ray bursts (LGRBs), a possible connection already pointed out by Tendulkar et al. (2017) and Marcote et al. (2017).

In Section 3 we review how the birth of a millisecond magnetar has been proposed as the central engine of both long LGRBs (Usov 1992; Wheeler et al. 2000; Thompson et al. 2004; Metzger et al. 2007, 2011) and SLSNe-I (Kasen \& Bildsten 2010; Woosley 2010). If such a magnetar is also capable of producing all of the observed features of FRB 121102, this places stringent constraints on the age of the system. In Section 3.2 we show the expanding oxygen-rich $\mathrm{SN}$ ejecta will become transparent to gigahertz emission on a timescale as short as a decade after the explosion. In Section 4 we describe possible sources for the quiescent radio counterpart within this picture on a timescale of decades after the explosion. These include emission escaping directly through the ejecta shell from a nascent, rotationally powered "magnetar wind nebula"; shock interaction between the fastest parts of the magnetar-boosted SN ejecta with the surrounding stellar progenitor wind; or an orphan radio afterglow from an initially off-axis LGRB. In Section 5 we discuss our results and expand on possible predictions of a connection between SLSNe-I/ LGRBs and FRBs. We briefly summarize our conclusions in Section 6.

\section{Connection to SLSNe-I and LGRBs}

It is now well established that both LGRBs and SLSNe-I exhibit a strong preference for low-mass, low-metallicity galaxies (e.g., Fruchter et al. 2006; Stanek et al. 2006; Modjaz et al. 2008; Castro Cerón et al. 2010; Levesque et al. 2010; Lunnan et al. 2014; Vergani et al. 2015; Japelj et al. 2016; Perley et al. 2016a, 2016b; Schulze et al. 2016). Marcote et al. (2017) and Tendulkar et al. (2017) noted that the host galaxy of FRB 121102 shares similar basic properties. Here we provide a detailed comparison.

In Figure 1 we directly compare the properties of the host of FRB 121102 to those of LGRBs and SLSN-I at $z \lesssim 1$. In particular, we show a comparison of the rest-frame optical 
absolute magnitudes, stellar masses, star formation rates, and metallicities. We find that in all of these fundamental properties, the host of FRB 121102 is remarkably similar to the hosts of LGRBs and SLSNe-I. It is located roughly at, or slightly below, the medians of the LGRB and SLSN-I host galaxy distributions, especially when considering the lowredshift population at $z \lesssim 0.3$.

Beyond their similar host galaxies, additional evidence connects SLSNe-I and LGRBs to similar stellar progenitors. Both classes tend to concentrate in bright UV regions of their hosts (e.g., Fruchter et al. 2006; Lunnan et al. 2014; Schulze et al. 2016). Moreover, despite clear spectroscopic differences between SLSNe-I and the LGRB-associated SNe near peak brightness (e.g., Liu \& Modjaz 2016), the nebular spectra (which probe the explosion ejecta) show close similarity (Nicholl et al. 2016). Thus, the differences between LGRB$\mathrm{SNe}$ and SLSNe-I may result from a difference in the engine properties, but the underlying progenitors are likely similar. The remarkable similarity of the host of FRB 121102 to those of LGRBs and SLSNe-I suggests that it too shared a similar progenitor and a common engine.

\section{Magnetar Birth in Core-collapse $\mathrm{SNe}$}

We now review the properties of millisecond magnetars on timescales well after the explosion, when they could be capable of producing a detectable FRB.

\subsection{Magnetars as FRB Sources}

Millisecond-period rotation at birth was long predicted to give rise to strong magnetic fields in neutron stars (e.g., Duncan \& Thompson 1992; Thompson \& Duncan 1993), even prior to the identification of Galactic magnetars (Kouveliotou et al. 1998). A neutron star with a surface dipole magnetic field strength of $B_{14}=B_{\mathrm{d}} / 10^{14} \mathrm{G}$ and birth spin period $P_{0}=1 P_{\mathrm{ms}}$ $\mathrm{ms}$ spins down due to magnetic torques, producing a spin-down luminosity $^{3}$

$$
\begin{aligned}
& L_{\mathrm{sd}}=5 \times 10^{46} B_{14}^{2} P_{\mathrm{ms}}^{-4}\left(1+\frac{t}{t_{\mathrm{sd}}}\right)^{-2} \mathrm{erg} \mathrm{s}^{-1} \\
& \underset{t \gg t_{\mathrm{sd}}}{\approx} 8 \times 10^{40} B_{14}^{-2} t_{10}^{-2} \mathrm{erg} \mathrm{s}^{-1},
\end{aligned}
$$

such that its spin period increases with time as

$$
P=P_{0}\left(1+\frac{t}{t_{\mathrm{sd}}}\right)^{1 / 2} \underset{t \gg t_{\mathrm{sd}}}{\approx} 27 \mathrm{~ms} B_{14} t_{10}^{1 / 2},
$$

where the spin-down timescale is given by

$$
t_{\mathrm{sd}} \simeq 4.7 \text { day } B_{14}^{-2} P_{\mathrm{ms}}^{2}
$$

We have normalized time $t=10 t_{10} \mathrm{yr}$ to a decade following the explosion, and in the final equality of Equations (1) and (2) we have assumed late-times $t \gg t_{\text {sd }}$.

The rotational energy of a magnetar has been suggested as a power source for both LGRB jets (e.g., Usov 1992; Wheeler et al. 2000; Thompson et al. 2004; Bucciantini et al. 2008; Metzger et al. 2011) and SLSNe-I (e.g., Kasen \& Bildsten

\footnotetext{
3 We have adopted the vacuum dipole spin-down convention employed by Kasen \& Bildsten (2010), which however differs in normalization from the force-free spin-down rate, which is likely more applicable in the plasma-dense environment of a young neutron star (e.g., Spitkovsky 2006).
}

2010; Woosley 2010; Metzger et al. 2015). Powering the optical light curve of SLSNe-I requires that the time $t_{\mathrm{sd}}$ over which the rotational energy is extracted be comparable to, or moderately less than, the timescale of the SN optical peak of weeks. Model fits to SLSN-I light curves generally find values of $B_{14} \sim 1-10$ and $P \sim 3-5 \mathrm{~ms}$ (e.g., Chatzopoulos et al. 2013; Nicholl et al. 2014). By contrast, powering the relativistic jet of an LGRB typically requires $P \sim 1-2 \mathrm{~ms}$ and a much stronger field $B_{14} \gtrsim 10-30$ in order to match the spin-down luminosity to the beaming-corrected LGRB luminosities of $\sim 10^{50} \mathrm{erg} \mathrm{s}^{-1}$. However, because of the short timescale of the engine, the light curves of LGRB-SN themselves are powered primarily by radioactive ${ }^{56} \mathrm{Ni}$ (e.g., Metzger et al. 2015, Cano et al. 2016). ${ }^{4}$

We now review possible ways that a young magnetar could power FRBs through its rotational or magnetic energy, focusing on constraints on the age of the system to explain FRB 121102. Each radio burst from FRB 121102 carries an energy $E_{\mathrm{FRB}}=f_{\mathrm{b}} E_{\mathrm{iso}}$, where $E_{\mathrm{iso}} \approx 10^{38}-10^{40} \mathrm{erg}$ is the measured isotropic equivalent energy (Chatterjee et al. 2017) and $f_{\mathrm{b}} \lesssim 1$ is the beaming fraction. Although the value of $f_{\mathrm{b}}$ is uncertain, it cannot be too small or the beaming-correct volumetric FRB rate would greatly exceed SLSNe-I/LGRB rates, even when accounting for the fact that a given magnetar-magnetar birth event can produce multiple FRBs (Section 5).

Therefore, if the radio bursts are powered by the magnetar rotational energy, the spin-down luminosity must exceed

$$
L_{\mathrm{sd}} \gtrsim L_{\mathrm{FRB}}=\frac{E_{\mathrm{FRB}}}{t_{\mathrm{FRB}}} \approx 10^{42} \mathrm{erg} \mathrm{s}^{-1} f_{\mathrm{b}}\left(\frac{E_{\mathrm{iso}}}{10^{39} \mathrm{erg}}\right)\left(\frac{t_{\mathrm{FRB}}}{1 \mathrm{~ms}}\right)^{-1},
$$

where $t_{\mathrm{FRB}}$ is the burst duration. Equation (1) shows that, even given a beaming fraction $f_{b} \ll 1$, powering an FRB through spin-down luminosity requires a very young pulsar/magnetar of age $t_{\text {age }} \lesssim 9\left(L_{\mathrm{FRB}} / 10^{41} \mathrm{erg} \mathrm{s}^{-1}\right)^{-1} B_{14}^{-1} \mathrm{yr}$ (see also Piro 2016; Lyutikov 2017), as well as an extremely efficient mechanism for converting spin-down power into coherent radio emission. Rotational energy is furthermore not viable as the FRB power source for a magnetar capable of powering a normal LGRB with $B_{14} \gtrsim 10$, since this would require an age much less than the duration of four years over which FRB 121102 has been observed to burst.

Alternatively, radio bursts may be powered by the release of magnetic energy, similar to but potentially more extreme than giant flares produced by Galactic magnetars (Giannios 2010; Popov \& Postnov 2013; Thornton et al. 2013; Lyubarsky 2014). Lyubarsky (2014) provides an explicit model for how a magnetic pulse interacting with the magnetar's rotation-powered wind nebula could give rise to an FRB-like burst through synchrotron maser instability. Assuming an internal magnetic field strength of $B_{\text {int }} \approx 10^{16} \mathrm{G}$, similar to that expected in the remnants of magnetars at birth (e.g., Mösta et al. 2015), the internal magnetic energy is $E_{\mathrm{B}} \approx\left(4 \pi R_{\mathrm{ns}}^{3} / 3\right)\left(B_{\text {int }}^{2} / 8 \pi\right) \approx 3 \times 10^{49}\left(B_{\text {int }} / 10^{16} \mathrm{G}\right)^{2} \mathrm{erg}$, where $R_{\mathrm{ns}}=12 \mathrm{~km}$ is the neutron star radius. A given magnetar

\footnotetext{
4 The ultra-long GRB111209 (engine duration $\approx 1$ hour) was accompanied by an unusually luminous (though not quite super-luminous) SN 2011kl (Greiner et al. 2015); this event could represent a hybrid event with an intermediateduration engine that produced both a successful jet and later contributed to powering the SN emission (Metzger et al. 2015).
} 
could therefore produce a maximum number of bursts given by

$$
\begin{aligned}
N_{\mathrm{FRB}} & =\frac{E_{\mathrm{B}}}{E_{\mathrm{FRB}}} \\
& \approx 3 \times 10^{2} f_{b}^{-1}\left(\frac{f_{\mathrm{r}}}{10^{-8}}\right)\left(\frac{B_{\mathrm{int}}}{10^{16} \mathrm{G}}\right)^{2}\left(\frac{E_{\mathrm{FRB}}}{10^{39} \mathrm{erg}}\right)^{-1},
\end{aligned}
$$

where $f_{\mathrm{r}}$ is the fraction of the flare energy placed into coherent radio emission.

We have normalized the FRB efficiency to a value of $f_{\mathrm{r}} \approx 10^{-8}$, as was estimated by Lyubarsky (2014) for a nebula with an electron/positron density of $n_{ \pm} \approx 10^{-6} \mathrm{~cm}^{-3}$. However, this efficiency scales approximately linearly with the pair density in his model and thus could in principle be much higher given the expected range of pair densities $n_{+} \sim 10^{-7}-10^{-3}$ $\mathrm{cm}^{-3}$ in the young magnetar nebulae on timescales of decades (depending on the pair multiplicity of the wind; see Equation 14). On the other hand, searches for a coincident radio counterpart to the giant flare from the Galactic magnetar SGR 1806-20 ruled out radio emission similar to the observed bursts from FRB 121102 (Tendulkar et al. 2016), potentially implying a lower radiative efficiency. Spitler et al. (2014) observed 11 bursts from FRB 121102 in about 0.6 days of total observing time with an average isotropic energy fluence per burst of $\approx 4 \times 10^{38} \mathrm{erg}$; extended over the $\gtrsim 4 \mathrm{yr}$ period of burst activity, this suggests a total number of bursts of $\gtrsim 2 \times 10^{4}$ bursts for $f_{b} \sim 1$, already comparable to the estimate of $N_{\text {FRB }}$ from Equation (5). Energetic constraints may therefore be pointing to a system lifetime not much longer than the current bursting duration, although this obviously depends on the uncertain values of $f_{b}$ and $f_{\mathrm{r}}$.

If the sources of the radio bursts were in fact giant magnetic flares, we might expect to detect gamma-ray emission coincident with the bursts from FRB 121102, of luminosity $L_{\gamma} \sim 10^{47} \mathrm{erg} \mathrm{s}^{-1}$, similar to giant flares in our galaxy (e.g., Palmer et al. 2005). However, this emission would be too dim to detect at the distance of FRB 121102 by Fermi GBM or Swift BAT. Even if the young magnetar produced quiescent X-ray emission above the Chandra upper limits of $\sim 5 \times 10^{41} \mathrm{erg} \mathrm{s}^{-1}$, the oxygen-rich $\mathrm{SN}$ ejecta will likely remain opaque to photoelectric (bound-free) absorption on timescales of centuries or longer after the explosion (Appendix, Equation (36)).

\subsection{Transparency of the SN Ejecta}

Additional constraints on the age of the system result from requiring that the $\mathrm{GHz}$ radio emission be able to escape through the expanding $\mathrm{SN}$ ejecta, as well as the requirement to not overproduce the maximum local contribution to the DM or its derivative (see also, e.g., Connor et al. 2016; Piro 2016).

We consider the stellar progenitor to be a compact, strippedenvelope star, similar to those of LGRBs and SLSNe-I. We adopt characteristic values of $M_{\mathrm{ej}} \approx 10 M_{\odot}$ and $v_{\mathrm{ej}} \approx 10^{4}$ $\mathrm{km} \mathrm{s}^{-1}$ for the mass and mean velocity of the ejecta, motivated by observations of SN associated with LGRBs and SLSNe-I (e.g., Nicholl et al. 2015). These fiducial values imply a kinetic energy of $\approx M_{\mathrm{ej}} v_{\mathrm{ej}}^{2} / 2 \approx 10^{52} \mathrm{erg}$, similar to those of hyperenergetic LGRB-SN and some SLSNe-I.

The free expansion phase of the $\mathrm{SN}$ blast wave lasts until it sweeps up a mass comparable to its own in the pre-explosion stellar wind or interstellar medium. The density profile of a steady wind is given by $\rho_{\mathrm{w}}=\dot{M}_{\mathrm{w}} /\left(4 \pi r^{2} v_{\mathrm{w}}\right)=A / r^{2}$, where $\dot{M}_{\mathrm{w}}$ is the wind mass-loss rate and $v_{\mathrm{w}} \approx 1000 \mathrm{~km} \mathrm{~s}^{-1}$ is the wind velocity

of the compact progenitor star. We normalize the wind parameter $A=\dot{M}_{\mathrm{w}} /\left(4 \pi v_{\mathrm{w}}\right)$ according to the standard convention (e.g. Chevalier \& Li 1999),

$$
A_{\star} \equiv \frac{A}{5 \times 10^{11} \mathrm{~g} \mathrm{~cm}^{-1}} \approx 1.0\left(\frac{\dot{M}_{w}}{10^{-5} M_{\odot} \mathrm{yr}^{-1}}\right)\left(\frac{10^{3} \mathrm{~km} \mathrm{~s}^{-1}}{v_{\mathrm{w}}}\right)
$$

and thus consider values $A_{\star} \sim 0.1-10$, given the uncertain mass-loss history prior to the explosion. A typical ejecta will slow down once it reaches the deceleration radius $R_{\mathrm{dec}}=M_{\mathrm{ej}} /(4 \pi A)$ at which its swept up mass equals its own; this occurs on a timescale of ${ }^{5}$

$$
t_{\mathrm{dec}}=\frac{R_{\mathrm{dec}}}{v_{\mathrm{ej}}}=\frac{M_{\mathrm{ej}}}{4 \pi A v_{\mathrm{ej}}} \approx 10^{5} \mathrm{yr} A_{\star}^{-1} M_{10} v_{9}^{-1},
$$

where $M_{10} \equiv M_{\mathrm{ej}} / 10 M_{\odot}$ and $v_{9} \equiv v_{\mathrm{ej}} / 10^{4} \mathrm{~km} \mathrm{~s}^{-1}$. Thus, at times $t \ll t_{\mathrm{dec}}$ the mean ejecta radius increases as

$$
R_{\mathrm{ej}}=v_{\mathrm{ej}} t \approx 0.11 \mathrm{pc} v_{9} t_{10}
$$

and hence will remain smaller than the $5 \mathrm{GHz}$ VLBI upper limit on the quiescent radio source FRB 121102 of $\lesssim 0.7$ pc (Marcote et al. 2017) for $t \lesssim 70 \mathrm{yr}$ for typical parameters.

On timescales exceeding substantial rotational energy input from the magnetar $\left(t \gg t_{\mathrm{sd}}\right.$; Equation (3)), the ejecta will approach homologous expansion with a mean density decreasing approximately as $\rho_{\mathrm{ej}}=\left(3 M_{\mathrm{ej}} / 4 \pi R_{\mathrm{ej}}^{3}\right) \propto t^{-3}$. The corresponding mean free electron density is

$$
n_{\mathrm{e}} \simeq \frac{3 M_{\mathrm{ej}} f_{\mathrm{ion}}}{8 \pi R_{\mathrm{ej}}^{3} m_{p}}=4.5 \times 10^{4} \mathrm{~cm}^{-3} f_{\text {ion }} M_{1} v_{9}^{-3} t_{10}^{-3},
$$

where $f_{\text {ion }}$ is the ionized fraction of the ejecta, which in general will vary with radius out through the ejecta (see below). Based on the anticipated nucleosynthesis in engine-driven $\mathrm{SNe}$ (e.g., Maeda et al. 2002), we assume for simplicity that the ejecta are composed entirely of oxygen, though in detail they will contain smaller mass fractions of other elements with mass-to-charge ratios $A / Z=2$ like helium, carbon, and iron.

The resulting plasma frequency within the ejecta is

$$
\nu_{\mathrm{p}}=\frac{1}{2 \pi} \sqrt{\frac{4 \pi n_{\mathrm{e}} e^{2}}{m_{e}}} \simeq 0.06 \mathrm{GHz} f_{\text {ion }}^{1 / 2} M_{10}^{1 / 2} v_{9}^{-3 / 2} t_{10}^{-3 / 2} .
$$

Hence, even for $f_{\text {ion }} \approx 1$, there is no significant barrier to the escape of gigahertz radiation a few years after the explosion.

A more stringent constraint on the escape of radio emission from the nebula comes from the free-free optical depth for our assumed oxygen-dominated composition. Absent external radiation, the expanding $\mathrm{SN}$ ejecta will cool adiabatically, becoming almost completely neutral $\left(f_{\text {ion }} \ll 1\right)$ once the temperature drops below a few thousand kelvins on a timescale of months after the explosion. However, the ejecta eventually becomes reionized by two processes. First, a reverse shock is produced as the SN ejecta decelerates upon colliding with the progenitor wind, with a high enough temperature to completely

\footnotetext{
5 In reality, the steady wind profile will become invalid well before $R_{\mathrm{dec}}$, but this doesn't affect our subsequent conclusions.
} 


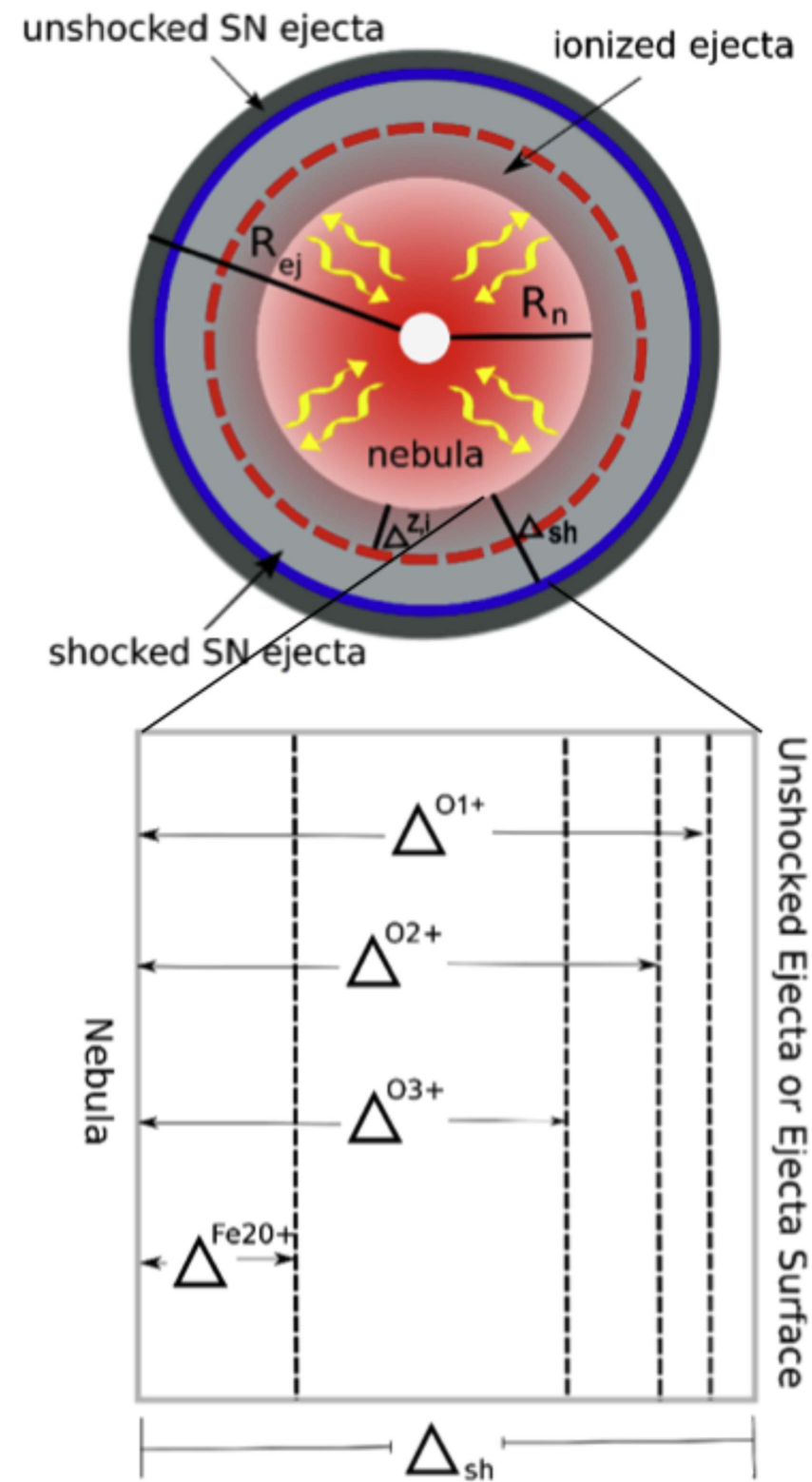

Figure 2. Schematic illustration of the magnetar nebula embedded in the expanding SN ejecta and the ionization structure (adapted from a similar figure in Metzger et al. 2014).

reionize the outer layers of the ejecta (Piro 2016). A second potential source of ionization comes from the central pulsar/ magnetar nebula, which provides a luminous source of UV/X-ray radiation, which acts to reionize the ejecta over time from within (Metzger et al. 2014). This produces multiple ionization fronts, such that $f_{\text {ion }}$ will decrease with radius from the inner edge of the ejecta directly exposed to the nebula out to the surface (Figure 2).

If the nebula produces continuum radiation with comparable energy across a range of frequencies from soft UV to soft $\mathrm{X}$-rays, then, in general, low ionization states (e.g., O I, O II) with threshold ionization frequencies in the UV are easier to photoionize than higher states (e.g., O VII, O VIII) with ionization frequencies at soft X-rays, due in part to the greater abundance of ionizing photons at lower energies. In the Appendix we estimate that the nebula may completely ionize $\mathrm{O}$ I and $\mathrm{O}$ II on a timescale of a decade or less, depending sensitively on the ejecta mass and the magnetic field of the magnetar. However, complete ionization (up to O VIII) probably requires centuries or longer, depending sensitively on the ejecta mass and the magnetar field. One implication of this is that soft X-rays are probably still trapped within the ejecta, consistent with the Chandra nondetection of FRB 121102 reported by Chatterjee et al. (2017) (see Equation (36) in the Appendix). This analysis also suggests that, on timescales of the few decades of interest, the radius- or massaveraged ionized fraction will range from $f_{\text {ion }} \approx 0$ (if the ejecta remains entirely neutral) to $f_{\text {ion }} \approx 3 / Z=0.4$ if O I-O III are ionized. ${ }^{6}$

The free-free optical depth through the ejecta shell for an oxygen-dominated composition $(Z=8, A=16)$ is

$$
\begin{aligned}
\tau_{\mathrm{ff}} & =\left(0.018 Z^{2} \nu^{-2} T_{\mathrm{ej}}^{-3 / 2} n_{\mathrm{e}} n_{\mathrm{ion}} \bar{g}_{\mathrm{ff}}\right) R_{\mathrm{ej}} \\
& \approx 93 \bar{g}_{\mathrm{ff}} f_{\text {ion }}^{2} \nu_{\mathrm{GHz}}^{-2} T_{4}^{-3 / 2} M_{10}^{2} t_{10}^{-5} v_{9}^{-5},
\end{aligned}
$$

where $n_{\text {ion }} \approx 2 n_{\mathrm{e}} / A$ is the ion density, $T_{\mathrm{ej}}=10^{4} T_{4} \mathrm{~K}$ is the ejecta temperature normalized to a typical value for photoionized gas and $\bar{g}_{\mathrm{ff}} \sim 1$ is the Gaunt factor.

An even more stringent constraint comes from the DM through the ejecta shell,

$$
\mathrm{DM}_{\mathrm{ej}} \simeq n_{\mathrm{e}} R_{\mathrm{ej}} \approx 462 \mathrm{pc} \mathrm{cm}^{-3}\left(\frac{f_{\text {ion }}}{0.1}\right) M_{10} v_{9}^{-2} t_{10}^{-2},
$$

which at a minimum must be less than the local contribution of $\mathrm{DM}_{\text {local }}<\mathrm{DM}_{\text {host+local }} \approx 55-225 \mathrm{pc} \mathrm{cm}^{-3}$ (Tendulkar et al. 2017). Another related constraint comes from the time derivative of the dispersion measure,

$$
\left|\frac{d \mathrm{DM}_{\mathrm{ej}}}{d t}\right|=\frac{2 \mathrm{DM}_{\mathrm{ej}}}{t} \approx 92 \mathrm{pc} \mathrm{cm}^{-3} \mathrm{yr}^{-1}\left(\frac{f_{\text {ion }}}{0.1}\right) M_{10} v_{9}^{-2} t_{10}^{-3},
$$

which Piro (2016) estimate obeys $d \mathrm{DM}_{\mathrm{ej}} / d t \lesssim 2 \mathrm{pc} \mathrm{cm}^{-3} \mathrm{yr}^{-1}$ for FRB 121102.

If just $\mathrm{O}$ I is ionized, then $f_{\text {ion }} \approx 0.1$ and both constraints result in a similar minimum source age of $t \gtrsim 30 \mathrm{yr}$ for fiducial parameters. By contrast, if O I remains neutral because the UV radiation field of the nebula is weak, then the age could be younger, while if $\mathrm{O}$ II is ionized the minimum age could approach a century.

Using our oxygen ionization model developed in the Appendix, Figure 3 compares the time evolution of DM and $d \mathrm{DM} / d t$ for different assumptions about the magnetar field strength, SN ejecta mass, and the fraction of the luminosity of the magnetar wind nebula in UV ionizing photons, $\epsilon_{\text {ion }}$. Whether the source age constraint is closer to 10 or 100 years old requires a more detailed model for the ionization structure of the ejecta, including a more accurate model for the SED of the magnetar wind nebula.

\section{Source of Quiescent Synchrotron Radio Emission}

We consider three possible sources for the quiescent synchrotron radio source, each potentially expected on decade to century timescales following the birth of a millisecond magnetar. These are (1) emission from the rotationally powered

\footnotetext{
6 A high mass fraction of helium, which will ionize at the same time as $\mathrm{O}$ and O II (Metzger et al. 2014), could also contribute to a larger value of $f_{\text {ion }}$.
} 

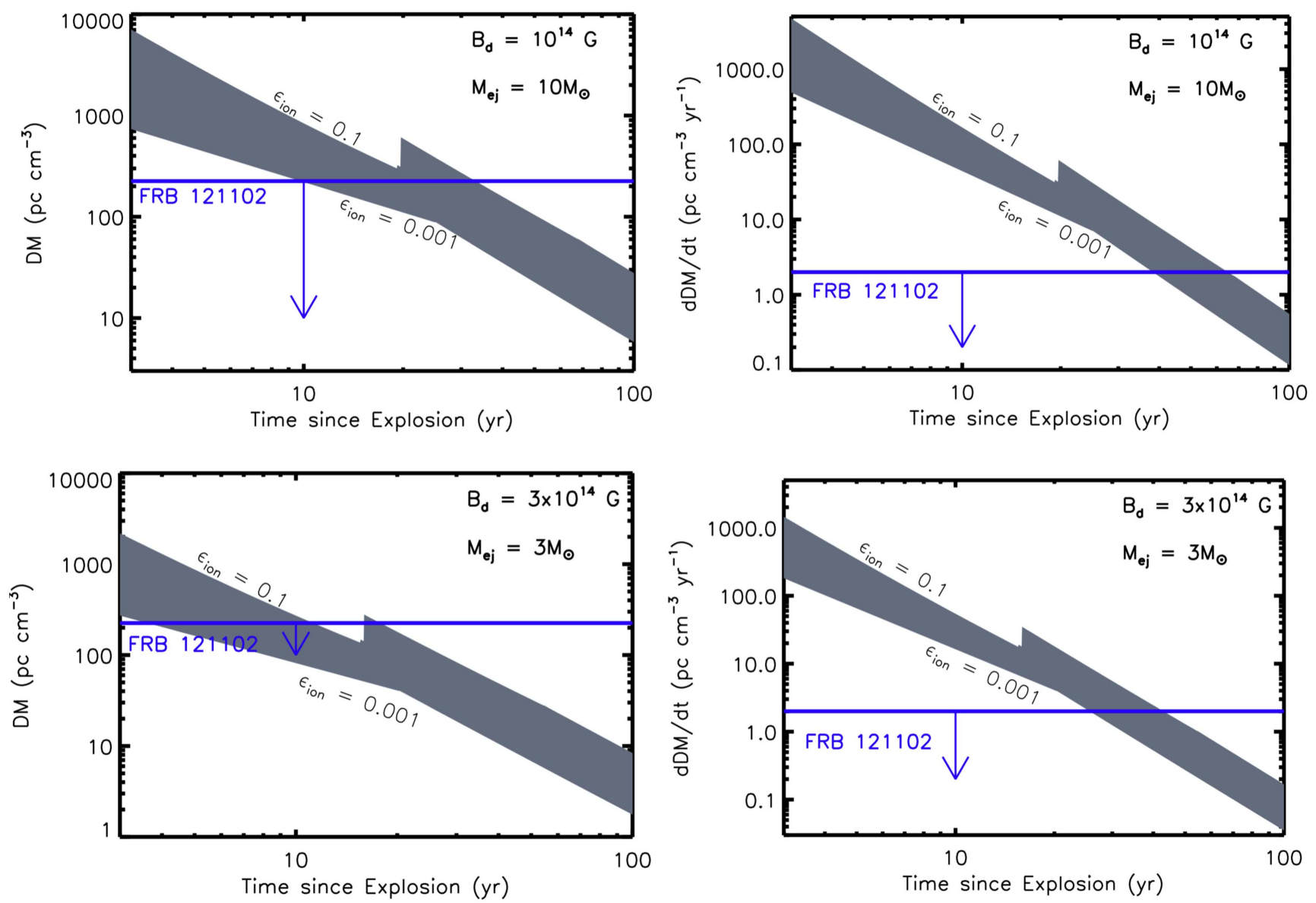

Figure 3. Gray region shows the dispersion measure (DM; left panels) and its time derivative (right panels) through the expanding oxygen-rich SN ejecta as a function of time since the explosion across a range of values for the fraction $\epsilon_{\text {ion }}=0.001-0.1$ of the magnetar luminosity placed into ionizing UV radiation. We have assumed an ejecta velocity of $\nu_{\mathrm{ej}}=10^{9} \mathrm{~cm} \mathrm{~s}^{-1}$ and an electron temperature in the ionized layer of $T=10^{4} \mathrm{~K}$ (see the Appendix). The top and bottom panels are shown for different values of the magnetar dipole field strength $B_{\mathrm{d}}=10^{14}, 3 \times 10^{14} \mathrm{G}$, and total SN ejecta mass $M_{\mathrm{ej}}=3,10 M_{\odot}$, respectively.

magnetar wind nebula (Section 4.1); (2) shock interaction between the blast wave and the external wind of the progenitor star (Section 4.2); and (3) an orphan afterglow from an initially off-axis LGRB that accompanied the core-collapse event (Section 4.3).

We first note a general constraint on the size of the radioemitting region, $R_{\text {rad, }}$, which is set by the brightness temperature:

$$
T_{\mathrm{B}}=\frac{c^{2} D^{2} F_{\nu}}{2 \pi k R_{\mathrm{rad}}^{2} \nu^{2}} \approx 2 \times 10^{9} \mathrm{~K}\left(\frac{F_{\nu}}{250 \mu \mathrm{Jy}}\right) \nu_{\mathrm{GHz}}^{-2}\left(\frac{R_{\mathrm{rad}}}{1 \mathrm{pc}}\right)^{-2} .
$$

The observed radio spectrum shows a flat spectral index $\beta \approx-0.2$ at frequencies $\gtrsim 1.6 \mathrm{GHz}$ (Chatterjee et al. 2017). However, synchrotron self-absorption from mildly relativistic electrons should instead produce a rising spectrum with $\beta \approx 2-2.5$ for brightness temperatures exceeding the "temperature" of mildly relativistic electrons of Lorentz factor $\gamma_{e} \sim 10$ contributing to the radio emission in this range, e.g., $T_{\mathrm{B}} \gtrsim \gamma_{e} m_{e} c^{2} / k \approx 5 \times 10^{10}\left(\gamma_{e} / 10\right) \mathrm{K}$. The observed $1.6 \mathrm{GHz}$ flux of $F_{\nu} \approx 250 \mu \mathrm{Jy}$ therefore requires an emitting size

$$
R_{\text {rad }} \gtrsim 0.2\left(\gamma_{e} / 10\right)^{-1 / 2} \mathrm{pc} \text {. }
$$

This is consistent with the $5 \mathrm{GHz}$ VLBI size constraint $R_{\text {rad }} \lesssim 0.7$ pc (Marcote et al. 2017). However, on fairly generic grounds, this does suggest that the quiescent radio source should possess a self-absorption break not too far below the gigahertz band.

Likewise, if the radio bursts must pass through the emitting region of the quiescent source in escaping the environment of the burst, as is the case in all of our proposed scenarios, then its low-frequency spectrum could also experience significant synchrotron self-absorption (see also Yang et al. 2016), additionally helping to explain the lack of low-frequency FRB radio detections (e.g., Karastergiou et al. 2015; Caleb et al. 2016; Rowlinson et al. 2016).

\subsection{Magnetar Wind Nebula}

As in other young pulsar wind nebulae (Gaensler \& Slane 2006), the pulsar wind from the magnetar inflates a nearly spherical bubble of relativistic electron/positron pairs behind the SN ejecta of characteristic radius $R_{\mathrm{n}} \lesssim R_{\mathrm{ej}}$ and volume $V_{\mathrm{n}} \approx 4 \pi R_{\text {neb }}^{3} / 3$ (Metzger et al. 2014; Murase et al. 2016). In principle, the quiescent radio source could therefore represent synchrotron radiation from this nebula (if the ejecta is transparent to free-free absorption to a burst at gigahertz frequencies, it will necessarily be transparent at higher frequencies). A relatively flat radio spectrum is also a common observational feature of $\mathrm{PWNe}$; 
for instance, $\beta \approx-0.25$ in the Crab Nebula (Bietenholz et al. 1997 ) is very similar to that observed in the lower frequency bands of FRB 121102.

We can estimate the magnetic field of the nebula at time $t$ crudely by assuming that the magnetic energy $\left(B_{\mathrm{n}}^{2} / 8 \pi\right) V_{\mathrm{n}}$ is a fraction $\epsilon_{B}$ of the injected spin-down energy $\sim L_{\mathrm{sd}} t$ :

$$
B_{\mathrm{n}} \simeq\left(\frac{6 \epsilon_{B} L_{\mathrm{sd}} t}{R_{\mathrm{n}}^{3}}\right)^{1 / 2} \simeq 7 \times 10^{-3} \mathrm{G} \epsilon_{B,-2}^{1 / 2} t_{10}^{-2} B_{14}^{-1 / 2} v_{9}^{-3 / 2},
$$

where we have taken $R_{\mathrm{n}} \approx R_{\mathrm{ej}}$ and have normalized $\epsilon_{B}=10^{-2} \epsilon_{B,-2}$ to a value similar to the magnetization inferred for the Crab Nebula (e.g., Kennel \& Coroniti 1984).

There are several locations within the nebula where electrons could be accelerated to relativistic velocities. One source of particle acceleration is the wind termination shock (Kennel \& Coroniti 1984). Electron/positron pairs are carried out by the pulsar wind at a rate $\dot{N}_{ \pm}=\mu_{ \pm} \dot{N}_{\mathrm{GJ}}$, where $\dot{N}_{\mathrm{GJ}}=$ $8 \pi^{2} B_{\mathrm{d}} P^{-2} R_{\mathrm{NS}}^{3} / e c$ is the Goldreich-Julian flux and $\mu_{t}$ is the pair multiplicity. The number density of freshly-injected in the nebula is thus approximately

$$
n_{ \pm} \simeq \frac{\dot{N}_{ \pm} t}{V_{\mathrm{n}}} \approx 1.3 \times 10^{-5} \mathrm{~cm}^{-3}\left(\frac{\mu_{ \pm}}{10^{2}}\right) B_{14}^{1 / 5} M_{1}^{3 / 5} v_{9}^{-9 / 5} t_{1}^{-7 / 5}
$$

where we have normalized the pair multiplicity to value $\mu_{ \pm} \approx 10^{2}$ expected for young magnetar winds (e.g., Medin \& Lai 2010; Beloborodov 2013).

If these pairs are accelerated impulsively at the wind termination shock carrying most of the total spin-down luminosity, they will reach a random pair Lorentz

$$
\gamma_{ \pm} \simeq \frac{L_{\mathrm{sd}}}{\dot{N}_{ \pm} m_{e} c^{2}} \approx 7.5 \times 10^{8}\left(\frac{\mu_{ \pm}}{10^{2}}\right)^{-1} B_{14}^{-1} t_{1}^{-1} .
$$

However, the synchrotron frequency of such pairs,

$$
\begin{aligned}
h \nu_{\mathrm{m}} & =\frac{h}{2 \pi} \frac{e B_{\mathrm{n}} \gamma_{ \pm}^{2}}{m_{e} c} \\
& \approx 260 \mathrm{MeV}\left(\frac{\mu_{ \pm}}{10^{2}}\right)^{-2} M_{1}^{3 / 10} B_{14}^{-12 / 5} v_{9}^{-9 / 10} t_{1}^{-3.7}
\end{aligned}
$$

is typically in the X-ray to gamma-ray frequency range on timescales of decades to a century (depending on $\mu_{+}$), much too high to explain the observed $\mathrm{GHz}$ radio emission, unless the pair multiplicity is orders of magnitude higher than in wellobserved PWNe. Furthermore, extending a synchrotron spectrum $\nu F_{\nu} \propto \nu^{4 / 3}$ from the $\mathrm{UV} / \mathrm{X}$-ray to radio band produces a spectral index inconsistent with that measured from FRB 121102 and a flux which is much too low to explain the measured values.

However, radio emission from the Crab Nebula does not conform to the simplest picture in which all particle acceleration occurs due to diffusive shock acceleration at the pulsar wind termination shock. The observed radio frequency spectral index of $\beta \approx-0.25$ (Bietenholz et al. 2001) of optically thin synchrotron emission (across three decades in

\footnotetext{
7 Since $\dot{N}_{\mathrm{GJ}} \propto t^{-1}$ at times $t \gg t_{\mathrm{sd}}$, the number of pairs injected to the nebula is approximately the same per logarithmic time interval.
}

frequency) requires an energy distribution of the emitting electrons $d N / d E \propto E^{-p}$ with slope $p=2 \beta+1 \approx 1.5$. This "excess" of low energy electrons has been attributed to pairs ejected at a much earlier phase, when the spin-down power was higher, whose energy has been degraded by adiabatic expansion (Atoyan 1999). Such an excess could be produced in a very young magnetar remnant due to the much higher pair creation rate due to $\gamma-\gamma$ annihilation, as is expected less than months after the explosion while the compactness parameter of the nebula is still $\gg 1$, leading to a pair formation cascade (Metzger et al. 2014, Metzger \& Piro 2014). More detailed modeling is needed to determine whether the energy in currently slow-cooling relic pairs can explain the quiescent source.

On the other hand, observations of time variable "wisps" in the radio band of the Crab Nebula (Bietenholz et al. 2001) show that at least some radio-emitting electrons are being accelerated currently, in the same region as the higher energy emission. This has led to the suggestion of other acceleration sites than the termination shock, such as the magnetic reconnection in the striped pulsar wind (e.g., Sironi \& Spitkovsky 2011, Zrake \& Arons 2016), which particle-in-cell plasma simulations show can indeed produce flatter $(p<2)$ electron spectra (e.g., Sironi \& Spitkovsky 2014), consistent with that needed to explain a flatter spectral index $\beta \approx-0.2$.

We consider that such an anomalous particle distribution $p=$ 1.5 is also at work in young magnetar nebulae, and that it carries a majority of the pulsar power. For such a particle distribution $p<2$, most of the total energy is in high-energy electrons. Therefore, if the particle distribution extends to a maximum Lorentz factor $\gamma_{\mathrm{m}}$ with a corresponding maximum synchrotron frequency $\nu_{\mathrm{m}}$, the radio flux in the gigahertz band can be written as

$$
\begin{aligned}
F_{\nu} & \approx \frac{L_{\mathrm{sd}}}{4 \pi D^{2} \nu_{\mathrm{m}}}\left(\frac{\nu}{\nu_{\mathrm{m}}}\right)^{-0.25} \\
& \approx 70 \mu \mathrm{Jy} \nu_{\mathrm{GHz}}^{-0.25}\left(\frac{\nu_{\mathrm{m}}}{10^{13} \mathrm{~Hz}}\right)^{-0.75} B_{14}^{-2} t_{10}^{-2} .
\end{aligned}
$$

Thus, for $\nu_{\mathrm{m}} \lesssim 10^{13} \mathrm{~Hz}$ and $B_{14} \sim 1$, we can reach $1.7-10 \mathrm{GHz}$ radio fluxes comparable to the values $\approx 200 \mu \mathrm{Jy}$ measured from the quiescent counterparts of FRB 121102 on timescales of decades. For comparison, in the Crab Nebula, the value of $\nu_{\mathrm{m}}$ (occurring near the peak of the SED) occurs at $\nu_{\mathrm{m}} \approx 10^{14} \mathrm{~Hz}$. Equation (20) also makes clear that the nebula scenario is not a viable source of radio emission on decade timescales for magnetars with ultra-strong fields $\left(B_{14} \gtrsim 10\right)$, hypothesized to power LGRB jets, again because they spin down too quickly.

\subsection{External Blast Wave}

Another source of synchrotron radiation is shock interaction between the fastest layers of the SN ejecta and the surrounding wind of the progenitor star. The nebula inflated by the magnetar drives a shock through the ejecta, which reaches the surface of the star on a timescale comparable to the spin-down time $\sim t_{\mathrm{sd}}$ if the total rotational energy from the magnetar exceeds the initial kinetic energy of the ejecta of $\approx 10^{51} \mathrm{erg}$ (e.g., Chen et al. 2016; Kasen et al. 2016), that is, for magnetar birth spin periods of $P \lesssim 2-3 \mathrm{~ms}$. 
Two-dimensional hydrodynamical simulations of this process of bubble inflation by Suzuki \& Maeda (2016) show that final density distribution of the matter reaches a homologous state with the distribution of density with velocity given by $\rho(v) \propto v^{-\beta}$, where $\beta \approx 5-6$ in the outer ejecta, and $\beta \approx 0$ in an inner core of the ejecta (hereafter we adopt $\beta=6$ in the outer ejecta, and a flat $\beta=0$ inner core). The transition velocity between outer-inner ejecta profiles $v_{t}$ is therefore related to the total ejecta mass and energy by $v_{\mathrm{t}}=$ $\sqrt{10 E_{\mathrm{ej}} / 3 M_{\mathrm{ej}}}$. The ejecta energy distribution above $v_{\mathrm{t}}$ obeys $E_{>v}=\left(3 M_{\mathrm{ej}} v_{\mathrm{t}}^{2} / 4\right)\left(v / v_{\mathrm{ej}}\right)^{-1}$, and the adiabatic-shock dynamics are governed by energy conservation (e.g., Margalit \& Piran 2015)

$$
E_{>v} \approx M_{\mathrm{dec}}\left(R_{\mathrm{dec}}\right) v^{2}
$$

This implies a deceleration radius for a given mass layer to sweep up its own energy in the progenitor stellar wind

$$
R_{\mathrm{dec}}(v)=\frac{3 M_{\mathrm{ej}}}{16 \pi A}\left(\frac{v}{v_{\mathrm{t}}}\right)^{-3} .
$$

Modifying Equation (7), we find that the deceleration time for a mass layer at velocity $v$ is therefore

$$
t_{\mathrm{dec}}(v)=\frac{3 R_{\mathrm{dec}}}{4 v} \approx 5.7 \times 10^{4} \mathrm{yr} A_{\star}^{-1} M_{10}\left(v / v_{\mathrm{ej}}\right)^{-4} v_{9}^{-1},
$$

where the prefactor $3 / 4$ in the first equality results from solving the forward-shock dynamic equation. The layer undergoing deceleration at a given time $\left(t=t_{\mathrm{ST}}\right)$ has velocity

$$
v_{\mathrm{dec}}=8.7 \times 10^{9} \mathrm{~cm} \mathrm{~s}^{-1} t_{10}^{-1 / 4} A_{\star}^{-1 / 4} M_{10}^{1 / 4} v_{9}^{3 / 4} \text {, }
$$

mass

$$
\begin{aligned}
M_{\mathrm{dec}} & =4 \pi A R_{\mathrm{dec}} \\
\approx & 0.012 \quad M_{\odot} \quad t_{10}^{3 / 4} A_{\star}^{3 / 4} M_{10}^{1 / 4} v_{9}^{3 / 4},
\end{aligned}
$$

and kinetic energy

$$
E_{\mathrm{dec}}=E_{>v_{\mathrm{dec}}} \approx 1.7 \times 10^{51} \operatorname{erg} t_{10}^{1 / 4} A_{\star}^{1 / 4} M_{10}^{3 / 4} v_{9}^{9 / 4}
$$

$\nu \gtrsim 1.6 \mathrm{GHz}$ (Equation (12)) for timescales of decades and $A_{\star} \sim$ 10 (as needed to explain the observed radio fluxes; see below).

The shocked gas will accelerate relativistic electrons, with a characteristic Lorentz factor:

$$
\begin{aligned}
& \gamma_{m}=\epsilon_{e} \frac{m_{p}}{m_{e}} \frac{p-2}{p-1}\left(\frac{v_{\mathrm{dec}}}{c}\right)^{2} \\
& \underset{p=2.3}{\approx} 3.3 \epsilon_{e,-1} t_{10}^{-1 / 2} A_{\star}^{-1 / 2} M_{10}^{1 / 2} v_{9}^{3 / 2},
\end{aligned}
$$

where we have made the standard assumption of a shockaccelerated electron distribution $d N / d E \propto E^{-p}$ with $p \approx 2.3$ (characteristic of other trans-relativistic shocks) that carries a total fraction $\epsilon_{e}=0.1 \epsilon_{e,-1}$ of the shock power. The shockgenerated magnetic field, distributed throughout the shocked volume $\approx 4 \pi R_{\mathrm{dec}}^{3} / 3$, can be estimated as

$$
B=\left(\frac{6 \epsilon_{B} E_{\mathrm{dec}}}{R_{\mathrm{dec}}^{3}}\right)^{1 / 2} \approx 4.6 \times 10^{-3} \mathrm{G} \epsilon_{B,-1}^{1 / 2} t_{10}^{-1} A_{\star}^{1 / 2},
$$

where again $\epsilon_{B}=10^{-1} \epsilon_{B,-1}$ is the equipartition fraction. The resulting characteristic synchrotron frequency is

$$
\nu_{m}=\frac{1}{2 \pi} \frac{e B \gamma_{m}^{2}}{m_{e} c} \approx 1.61 \times 10^{5} \mathrm{~Hz} \epsilon_{e,-1}^{2} \epsilon_{B,-1}^{1 / 2} t_{1}^{-2} A_{\star}^{-1 / 2} M_{10} v_{9}^{3}
$$

while the flux density at the distance of FRB 121102 $\left(D=3 \times 10^{27} \mathrm{~cm}\right)$ is given by

$$
F_{\nu_{m}}=\frac{N_{e}}{4 \pi D^{2}} \frac{m_{e} c^{2} \sigma_{T}}{3 e} B \approx 9.9 \mathrm{mJy} \epsilon_{B,-1}^{1 / 2} t_{10}^{-1 / 4} A_{\star}^{5 / 4} M_{10}^{1 / 4} v_{9}^{3 / 4},
$$

where $N_{e}=M_{\mathrm{dec}} /\left(2 m_{p}\right)$ is the number of shocked electrons. The flux at higher frequencies $\nu>\nu_{\mathrm{m}}$ is given by

$$
\begin{aligned}
& F_{\nu}=F_{\nu_{m}}\left(\frac{\nu}{\nu_{m}}\right)^{-(p-1) / 2} \\
& \underset{p=2.3}{\approx} 34 \mu \mathrm{Jy} \nu_{\mathrm{GHz}}^{-0.65} \epsilon_{B,-1}^{0.83} \epsilon_{e,-1}^{1.3} t_{10}^{-1.55} A_{\star}^{0.925} M_{10}^{0.9} \nu_{9}^{2.7},
\end{aligned}
$$

where in the second line we have taken $p=2.3$. We thus see that its possible to reproduced the observed fluxes of $F_{\nu} \approx 200 \mu$ Jy in the $\mathrm{GHz}$ frequency range for $A_{\star} \sim 10$ and $\epsilon_{e} \approx 0.2$.

Finally, the synchrotron self-absorption frequency can be expressed as (Chevalier 1998)

$$
\begin{aligned}
& \nu_{\mathrm{a}} \approx 0.1 \mathrm{GHz} \xi(p) \epsilon_{e^{\frac{2 p-2}{p+4}}}^{\frac{p+2}{2(p+4)}} A_{\star}^{\frac{5-p}{2(p+4)}} M_{\mathrm{ej}}^{\frac{2 p-3}{2(p+4)}} v_{\mathrm{t}}^{\frac{6 p-9}{2(p+4)}} t^{-\frac{4 p+5}{2(p+4)}} \\
& \underset{p=2.3}{\approx} 0.14 \mathrm{GHz} \epsilon_{e,-1}^{0.41} \epsilon_{B,-1}^{0.34} A_{\star}^{0.21} M_{10}^{0.13} v_{9}^{0.38} t_{10}^{-1.13}
\end{aligned}
$$

Deceleration of this layer occurs on a radial scale

$$
R_{\mathrm{dec}}=\frac{4}{3} v_{\mathrm{dec}} t \approx 3.7 \times 10^{18} \mathrm{~cm} t_{10}^{3 / 4} A_{\star}^{-1 / 4} M_{10}^{1 / 4} v_{9}^{3 / 4},
$$

again marginally consistent with the upper limits on the $5 \mathrm{GHz}$ source size Marcote et al. (2017) and the lack of self-absorption at where $\xi(p)$ is an order unity prefactor. For $\epsilon_{e} \approx 0.2$ and $A_{\star} \sim 10$ we therefore expect the self-absorption frequency to fall marginally below the observational band, $\nu_{m}<\nu_{\mathrm{a}} \lesssim 1 \mathrm{GHz}$.

One apparent problem with this scenario is that the observed spectral index of $\beta \approx-0.2$ is flatter than the value $\beta=-0.65$ assumed here. If interpreted as optically thin synchrotron emission, $\beta \approx-0.2$ would appear to require $p \approx 2 \beta+1 \approx 1.4$, an 


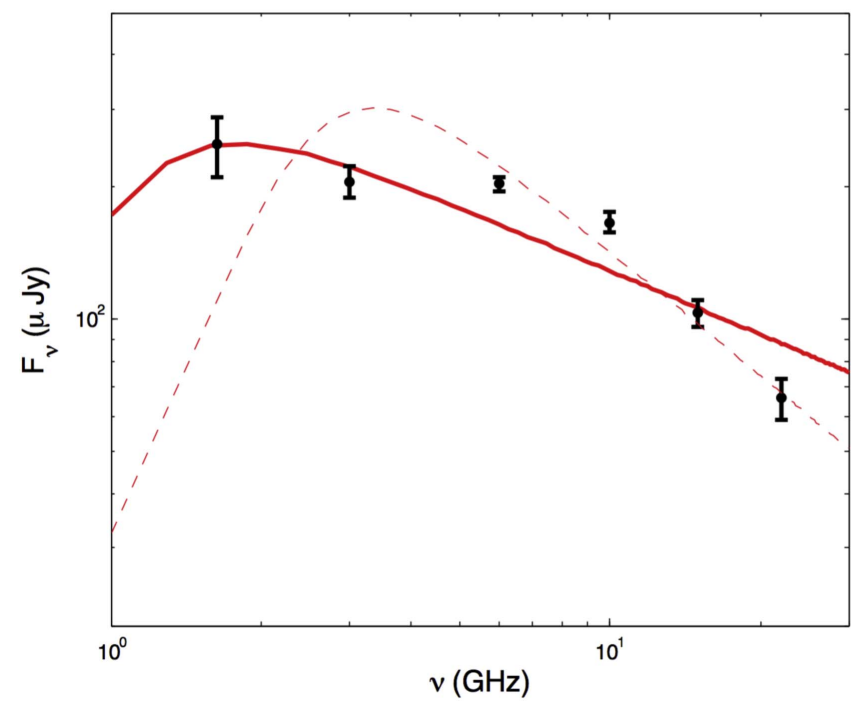

Figure 4. Spectrum of the quiescent radio source coincident with FRB 121102 (Chatterjee et al. 2017), compared to a model for a self-absorbed synchrotron spectrum of the form $F_{\nu}=F_{0}\left(\nu / \nu_{a}\right)^{5 / 2}\left(1-\exp \left[-\left(\nu / \nu_{a}\right)^{-(p+4) / 2}\right]\right)$ (Chevalier 1998), where $\nu_{a}$ is the self-absorption frequency and $p$ is the electron acceleration index. A solid line shows the best-fit model $\left(F_{0} \approx 350 \mu \mathrm{Jy}, \nu_{a}=1.2 \mathrm{GHz}, p=1.967\right)$, while a dashed line shows a "high- $p$ " model for which $F_{0}=460 \mu \mathrm{Jy}, \nu_{a}=2.9 \mathrm{GHz}$, and $p=2.9$.

electron distribution which as already discussed is extremely challenging to produce from particle acceleration at a shock. However, as discussed at the beginning of this section, synchrotron self-absorption could be setting in just below the frequency window of observations (Equation (15) and surrounding discussion). This implies that the true asymptotic spectral index in the frequency range of a few gigahertz could be steeper than the inferred value of $\beta \approx-0.2$; indeed, at the highest radio frequencies $\nu \gtrsim 10 \mathrm{GHz}$, there is indeed evidence for a steeper index closer to $\beta \approx-1$.

To illustrate this point, Figure 4 shows the spectrum of the quiescent radio source coincident with FRB 121102 (Chatterjee et al. 2017), compared to a model for a self-absorbed synchrotron spectrum of the form $F_{\nu}=F_{0}\left(\nu / \nu_{a}\right)^{5 / 2}$ $\left(1-\exp \left[-\left(\nu / \nu_{a}\right)^{-(p+4) / 2}\right]\right)$ from Chevalier (1998), where $\nu_{a}$ is the self-absorption frequency and $p$ is the electron acceleration index. A solid line shows the best-fit model $\left(F_{0} \approx 350 \mu \mathrm{Jy}\right.$, $\nu_{a}=1.2 \mathrm{GHz}, p=1.967$ ), while a dashed line shows a "high- $p$ " model for which $F_{0}=460 \mu \mathrm{Jy}, \nu_{a}=2.9 \mathrm{GHz}$, and $p=2.9$.

Another concern with this model is that radio emission has not yet been detected in coincidence with SLSNe-I, even in cases where relatively tight constraints are available, such as the nearby SLSN-I SN 2015bn $(z=0.1)$, for which upper limits on the 7.4 (22) $\mathrm{GHz}$ radio emission on a timescale of about one year were in the range $F_{\nu} \lesssim 40(75) \mu \mathrm{Jy}$ (Nicholl et al. 2016), corresponding to limits roughly four times deeper at the larger distance of FRB 121102. However, if the self-absorption frequency is indeed just below the $1 \mathrm{GHz}$ band on timescales of a few decades for FRB 121102, then on timescales of a year the synchotron selfabsorption (SSA) frequency would be higher, possibly in the $10-20 \mathrm{GHz}$ range, and hence the radio emission might be suppressed from the value predicted by Equation (30).

\subsection{Orphan Radio Afterglow from an Off-axis LGRB Jet}

Millisecond protomagnetars, along with black hole accretion, are contenders for the central engines of LGRBs (e.g., Thompson

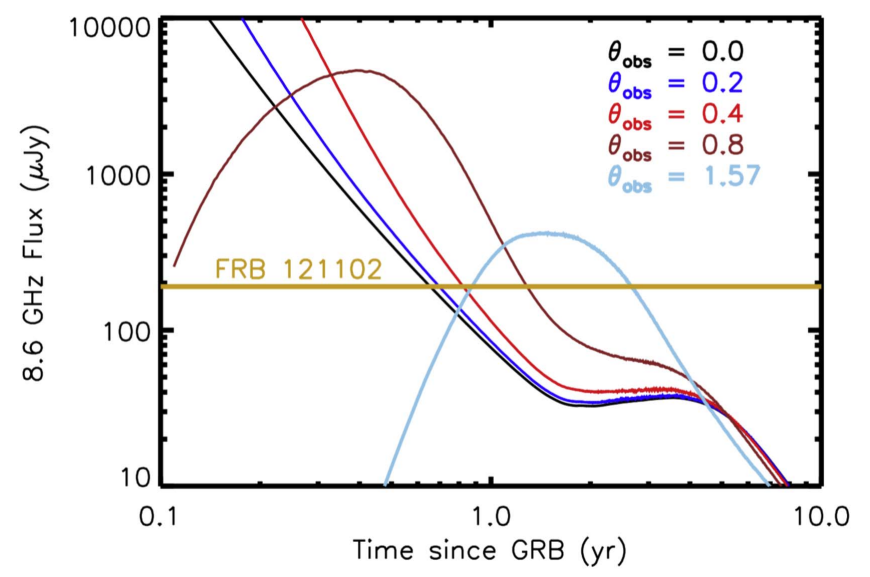

Figure 5. Model for off-axis orphan radio afterglow emission of a GRB jet for different viewing angles relative to the jet axis (van Eerten et al. 2010) compared to the flux density of FRB 121102. The jet kinetic energy is $E_{\mathrm{k}}=2 \times 10^{51} \mathrm{erg}$ and propagates into an external medium of constant density $n=1 \mathrm{~cm}^{-3}$. The parameters of the shock-accelerated electrons are $p=2.5$ and $\epsilon_{e}=\epsilon_{B}=0.1$.

et al. 2004). LGRB jets produce ultrarelativistic ejections in tightly collimated jets with opening angles $\theta_{j} \ll 1$ (Frail et al. 2001). Assuming that the radio bursts from FRB 121102 are isotropic (or at least not directed along the GRB jet direction), then the GRB jet would most likely be directed away from our line of sight. However, as material slows down by shocking the interstellar medium, even off-axis viewers enter the causal emission region of the synchrotron afterglow (Rhoads 1997). When viewed in an initial off-axis direction, the emission from such an "orphan afterglow" can at late times become approximately isotropic once the shocked matter decelerates to subrelativistic velocities (e.g., Zhang \& MacFadyen 2009; Wygoda et al. 2011).

Figure 5 compares the $8.6 \mathrm{GHz}$ off-axis LGRB afterglow models of van Eerten et al. (2010) for different viewing angles $\theta_{\mathrm{obs}}$ relative to the axis of the jet to the current flux of the quiescent radio source associated with FRB 121102 (gold line). The predicted off-axis jet emission drops below the emission from FRB 121102 for all viewing angles within roughly one year for moderately on-axis models $\theta_{\mathrm{ob}} \lesssim 0.8$ and on a timescale of about three years for the completely off-axis model $\theta_{\text {obs }}=1.57$. At face value, this would appear to disfavor the orphan afterglow explanation for the quiescent radio counterpart, since FRB 121102 has been undergoing bursts for at least about four years, placing an absolute lower limit on the time since the jet was launched. However, we note that the peak flux and peak time of the orphan afterglow depend sensitively on several uncertain parameters (jet energy, density of the external medium, electron acceleration efficiency $\epsilon_{e}$, and so on), such that in principle the radio flux could stay brighter much longer. For instance, the $4.9 \mathrm{GHz}$ flux of the nearby LGRB 030329 (at redshift $z=0.168$, similar to that of FRB 121102; Stanek et al. 2003; Hjorth et al. 2003) at $t \approx 10 \mathrm{yr}$ after the GRB was $F_{\nu} \approx 20 \mu \mathrm{Jy}$ (Mesler et al. 2012), somewhat brighter than predicted by the models in Figure 5 on a similar timescale, and it is decaying approximately as $F_{\nu} \propto t^{-1}$.

\section{Discussion}

Most of our proposed explanations for the quiescent radio source require a relatively young remnant, at most a few decades in age (or possibly less in the case of an off-axis LGRB). This can indeed be comparable to the timescale over which the oxygen-rich 
ejecta is becoming transparent to gigahertz radio emission, and over which the dispersion measure and its derivative decrease to values consistent with observations, although this depends sensitively on the degree to which the magnetar is able to ionize the ejecta (see the Appendix). Due to selection effects associated with the decaying rotational and magnetic energy of the magnetar likely causing the luminosity of bursts to also decay with time, we might expect that detected FRB sources will be dominated by those produced just as the ejecta is becoming optically thin to radiation. This also implies that a negative time derivative of the DM could be measured soon.

Such a scenario, in which we preferentially observe FRBs as soon as it becomes possible, is consistent with the lack of FRB discovery at subgigahertz frequencies (Karastergiou et al. 2015; Caleb et al. 2016; Rowlinson et al. 2016), since free-free absorption (e.g., Piro 2016) or synchrotron absorption from the same emission region responsible for the quiescent radio source (Equation (15) and surrounding discussion) becomes more severe at lower frequencies (Figure 4). Propagation of the pulse through the magnetar wind nebula might also induce excess Faraday rotation from that expected due to propagation through the Galaxy and the intergalactic medium, as measured by Masui et al. (2015) for FRB 110523 (Piro 2016).

A potential obstacle to a claimed association between FRBs and SLSNe-I or LGRBs are their relative rates. Howell et al. (2013) calculate the volumetric rate of SLSNe-I at $z \sim 1$ to be $91_{-36}^{+76} \mathrm{yr}^{-1} \mathrm{Gpc}^{-3}$ (see also McCrum et al. 2015). This is comparable to the estimated beaming-correct local $(z \approx 0)$ rate of LGRBs of $\approx 130_{-70}^{+60} \mathrm{Gpc}^{-3} \mathrm{yr}^{-1}$ for an assumed beaming fraction of $f_{\mathrm{b}}=1 / 100$ (Wanderman \& Piran 2010). These are substantially lower, by a factor of $\sim 10-100$, than the estimated volumetric FRB rate, using their observed rate of $\lesssim 10^{4}$ per sky per day of bursts $\gtrsim 1$ Jy and assuming distances derived assuming most of the excess DM is extragalactic (Thornton et al. 2013; Law et al. 2015; Rane et al. 2016; Scholz et al. 2016).

However, given the observation that some FRBs repeat (producing multiple bursts per object), the LGRB/SLSNe-I rate could well be compatible with the birth rate of FRB-producing objects. In such a scenario, most FRBs are repeating, but we only observe the very brightest ones, with FRB 121102 being an exception due to the high sensitivity of Areceibo. Furthermore, somewhat fine-tuned parameters are required in the magnetar model to give an extremely luminous SN (e.g., Kasen \& Bildsten 2010) or an LGRB (Metzger et al. 2011), implying that the true population of millisecond magnetar-forming $\mathrm{SNe}$ could be higher than estimated from these populations.

We now describe possible tests of the proposed SLSNe-I/ LGRB/FRB association. On several decade timescales after the explosion, the magnetar spin period will increase from an initial value of a few ms to $P \approx 30-300 \mathrm{~ms}$ (Equation (2)). Although no periodicities have been observed in the repeated bursts of FRB 121102 previously, this provides motivation to continue search for periodicities in this range. We might also expect to detect FRBs or their quiescent radio counterparts from the locations of previous LGRBs or SLSNe-I. ${ }^{8}$ Given that accurate localizations have only been available for a large number of

\footnotetext{
There have been previous suggestions connecting FRBs and LGRB (Deng \& Zhang 2014; Gao et al. 2014; Zhang 2014), but most of these are describing time coincidences. Time coincidence searches between FRBs and LGRBs (Yamasaki et al. 2016) led to the claimed discovery of a gamma-ray counterpart to FRB 131104 by DeLaunay et al. (2016). If this association is confirmed, there must be multiple classes of FRBs, including both "catastrophic" and "repeating" types.
}

GRBs since the launch of Swift in 2005, and that the first SLSNe-I were discovered in 2005 and 2006 (SN2005ap; Quimby et al. 2007, and SCP06F6; Barbary et al. 2009), the ability to monitor LGRB/SLSNe-I on $\gtrsim$ decade timescales is only now becoming possible. The known distance to the source could also aid such searches by reducing the possible range of DM. Higher frequency observations are preferred due to freefree absorption, especially in the case of searches for analogs to the quiescent source from FRB 121102.

Finally, in most of our scenarios we expect the quiescent radio source in FRB 121102 to fade on a timescale comparable to the inferred system age of decades or a century, such that $10 \%$ decay could be visible within a few years. Furthermore, at some point relatively soon a negative time derivative of the burst DM should be measured, though a more detailed model of the ionization evolution of the ejecta is required to better quantify this. Given the complex multidimensional structure of the interaction between the magnetar nebula and the ejecta and its susceptibility to hydrodynamic instabilities (e.g., RayleighTaylor fingers; Chen et al. 2016; Suzuki \& Maeda 2016) a complex, non-monotonic time evolution of the DM along a given line of sight might well be expected.

\section{Conclusions}

We propose that the association between the host galaxy of FRB 121102 and the properties of the hosts of SLSNe-I and LGRBs (Chatterjee et al. 2017; Tendulkar et al. 2017) can be understood if both classes of objects are associated with the birth of a millisecond magnetar. While the LGRBs and SLSNeI occur within minutes and weeks, respectively, of the core collapse, the escape of an FRB from the SN ejecta instead requires a timescale of a decade or longer. This timescale, which we argue is controlled mainly by photoionization by the magnetar wind nebula (see the Appendix), occurs somewhat earlier than in normal core-collapse SNe. This is in part because the ejecta velocities of magnetar-powered hypernovae can be about three times higher than those of normal core-collapse events and in part because an oxygen-rich ejecta is more challenging to ionize than a hydrogen-rich ejecta.

Following the submission of this paper, Kashiyama \& Murase (2017) proposed an association of FRB 121102 with a young pulsar embedded in the low mass ejecta of an "ultrastripped" hydrogen-poor supernova (e.g., Kleiser \& Kasen 2014, Tauris et al. 2015), a model that has been previously invoked to explain the observed population of fast-evolving Type Ic SNe (e.g., Drout et al. 2014). However, we note that the host galaxy properties of the ultra-fast stripped $\mathrm{SNe}$, such as their location in the mass-metallicity plane consistent with that comprising the bulk of star-forming galaxies, do not match those of the hosts of FRB 121102 or LGRBs/SLSNe-I (Drout et al. 2013, Drout et al. 2014), thus disfavoring such an association.

We have shown several ways in which the continuum radio source observed within a parsec of the location of FRB 121102 is naturally expected in the magnetar scenario, provided again that the source age is indeed at most a few decades old. These include emission from the magnetar wind nebula (in analogy with other PWNe like the Crab Nebula) or synchrotron emission from the fastest $\mathrm{SN}$ ejecta or from an off-axis LGRB afterglow. Distinguishing between these possibilities could be aided by further low-frequency observations of the bursts or quiescent sources, to look for evidence of free-free absorption 
and to ascertain whether both emission sources are passing through the same ejecta shell. Constraints on the flux and source size already imply that synchrotron self-absorption should become relevant at energies just below the current observing band.

Importantly, all of our proposed explanations for the quiescent radio source connect to an engine-powered explosion. An initially rapidly rotating (millisecond) magnetar or high-field pulsar is an inevitable feature the model to explain the quiescent radio source, even if the FRB pulses themselves are more likely powered by the dissipation of magnetic energy. Millisecond magnetars, perhaps produced preferentially in metal-poor environments like those characterizing the host of FRB 121102 , could conceivably be distinct in terms of their giant flare properties from the Galactic population of magnetars, which are mostly produced at solar metallicity or above.

Finally, we have suggested possible tests of the claimed association between FRBs an SLSNe-I by monitoring the locations of $\gtrsim$ decade old LGRBs and SLSN-I with arcsecond positions for coherent radio bursts and/or analogs to the quiescent radio source seen in association with FRB 121102. Measurement of the time derivative of the DM would tightly constrain the system age and is expected for a young source. Finally, the quiescent source should fade appreciably over the coming years to a decade.

B.D.M. gratefully acknowledges support from the National Science Foundation (AST-1410950, AST-1615084), NASA through the Astrophysics Theory Program (NNX16AB30G) and the Fermi Guest Investigator Program (NNX15AU77G, NNX16AR73G), the Research Corporation for Science Advancement Scialog Program (RCSA 23810), and the Alfred P. Sloan Foundation.

\section{Appendix \\ Photoionization of SN Ejecta by Magnetar Nebula}

In this section we estimate the conditions for the magnetar nebula to photo-ionize various atomic species through the ejecta shell as a function of time after the explosion, in order to assess the free-free opacity of the ejecta shell to radio emission and the photo-electric opacity to soft X-rays. For simplicity we consider that the ejecta is composed entirely of oxygen (Maeda et al. 2002).

A given atomic species $i$ will be ionized to a radial depth $\Delta_{\text {ion }}$ through the ejecta shell where the bound-free ${ }^{9}$ optical depth of a photon near the ionization threshold energy $h \nu_{\text {th }}$ reaches unity (Metzger et al. 2014; see Figure 2),

$$
\Delta_{\mathrm{ion}} \simeq \frac{2}{\rho_{\mathrm{ej}} \kappa_{\mathrm{bf}}\left(\nu_{\mathrm{th}}\right)}=\frac{2 A m_{p}}{\rho_{\mathrm{ej}} \sigma_{\mathrm{bf}} f_{\mathrm{n}}}
$$

where $\kappa_{\mathrm{bf}}=\left(f_{\mathrm{n}} \sigma_{\mathrm{bf}} / A m_{p}\right)$ is the bound-free opacity and

$$
\sigma_{\mathrm{bf}, \nu} \simeq \sigma_{\mathrm{th}}\left(\frac{\nu}{\nu_{\mathrm{th}}}\right)^{-3}, \nu \gtrsim \nu_{\mathrm{th}}
$$

is the bound-free cross section and $\sigma_{\text {th }}$ is the cross section at threshold. The factor of 2 in Equation (31) results because we have estimated the penetration depth as twice that of a photon

\footnotetext{
9 We neglect scattering opacity relative to absorptive opacity since at late times of interest the ejecta is optically thin to Thomson scattering.
}

with the threshold frequency $\nu=\nu_{\text {thr }}$ (see Appendix B of Metzger et al. 2014).

We assume that a fraction $\epsilon_{\text {ion }} \sim 0.001-0.1$ of the magnetar spin-down power $L_{\mathrm{sd}}$ (Equation (1)) is placed into the radiation energy distribution of the nebula $E_{\nu}$ near the threshold ionization frequency $\nu_{\text {th }}$, i.e. the ionization radiation energy density of the nebula incident on the ejecta is

$$
\nu_{\text {th }} u_{\text {ion }, \nu}=\epsilon_{\text {ion }} \frac{L_{\mathrm{sd}}}{4 \pi R_{\mathrm{ej}}^{2} c} .
$$

The "neutral" (non-ionized) fraction $f_{\mathrm{n}}$ of species $i$ in the layer directly exposed to the nebula is determined by the competition between the rates of photo-ionization and radiative recombination according to

$$
\begin{aligned}
f_{\mathrm{n}} & \simeq\left(1+\frac{4 \pi}{\alpha_{\mathrm{rec}} n_{\mathrm{e}}} \int \frac{J_{\nu}}{h \nu} \sigma_{\mathrm{bf}, \nu} d \nu\right)^{-1} \underset{f_{n} \ll 1}{\approx} \frac{\alpha_{\mathrm{rec}} M_{\mathrm{ej}} f_{\mathrm{ion}} h \nu_{\mathrm{th}}}{m_{p} \epsilon_{\mathrm{ion}} L_{\mathrm{sd}} t v_{\mathrm{ej}} \sigma_{\mathrm{th}}} \\
& \approx 7.6 \times 10^{-4}\left(\frac{0.1}{\epsilon_{\mathrm{ion}}}\right) f_{\mathrm{ion}} M_{10} B_{14}^{2} t_{10}\left(\frac{h \nu_{\mathrm{th}}}{10 \mathrm{eV}}\right) \frac{\alpha_{\mathrm{rec},-11}}{\sigma_{\mathrm{th},-18}}
\end{aligned}
$$

where $\sigma_{\text {th },-18}=\sigma_{\text {th }} /\left(10^{-18} \mathrm{~cm}^{2}\right), \alpha_{\text {rec }}=10^{-11} \alpha_{-11} \mathrm{~cm}^{3} \mathrm{~s}^{-1}$ is the rate of radiative recombination, $J_{\nu_{\mathrm{th}}}=c u_{\text {ion, } \nu} / 4 \pi$ is the mean intensity of the nebula near the ionization threshold incident on the ejecta of volume $V_{\mathrm{ej}} \simeq 4 \pi R_{\mathrm{ej}}^{3} / 3$.

On decade timescales of interest, Equation (34) in most cases we expect that $f_{\mathrm{n}} \ll 1$, in which case the thickness of the photo-ionized layer (Equation (31)) can be estimated as

$$
\begin{aligned}
\frac{\Delta_{\mathrm{ion}}}{R_{\mathrm{ej}}} \simeq & \frac{8 \pi A m_{p}^{2} \nu_{\mathrm{ej}} \epsilon_{\mathrm{ion}} L_{\mathrm{sd}} t R_{\mathrm{ej}}^{2}}{3 f_{\mathrm{ion}} M_{\mathrm{ej}}^{2} \alpha_{\mathrm{rec}}\left(h \nu_{\mathrm{th}}\right)} \\
& \approx 1.4\left(\frac{\epsilon_{\mathrm{ion}}}{0.1}\right) M_{10}^{-2} f_{\mathrm{ion}}^{-1} \alpha_{-11}^{-1} v_{9}^{3} t_{10} B_{14}^{-2}\left(\frac{h \nu_{\mathrm{th}}}{10 \mathrm{eV}}\right)^{-1} .
\end{aligned}
$$

A given species will thus become completely ionized throughout the ejecta once $\Delta_{\text {ion }} \approx R_{\mathrm{ej}}$, as occurs after a time:

$$
t_{\text {ion }} \approx 7.1 \mathrm{yr}\left(\frac{\epsilon_{\mathrm{ion}}}{0.1}\right)^{-1} M_{10}^{2} f_{\mathrm{ion}} \alpha_{-11} v_{9}^{-3} B_{14}^{2}\left(\frac{h \nu_{\mathrm{th}}}{10 \mathrm{eV}}\right) .
$$

Moving from O I to O VIII, threshold energies increase $h \nu_{\text {th }}=$ $[13.6,35,55,77,110,140,740,870] \mathrm{eV}$; the threshold cross sections are $\sigma_{\mathrm{th},-18}=[12,9,4,1.6,0.8,0.3,0.2,0.09]$ (Verner et al. 1996); and the recombination rates at $T \approx 10^{4}-3 \times 10^{4} \mathrm{~K}$ generally increase $\alpha_{-11}=[0.004-0.04,0.04-0.09,1.3-1.0,4.0-$ $3.0,0.5-1.5,0.9-0.3,3.6-1.7,4.6-2.2]$ (Nahar \& Pradhan 1997). Finally, the ionization fraction penetrating the $i$ th ionization layer will increase as $f_{\text {ion }}=i / 8$.

The net effect of the increasing values of $h \nu_{\text {th }}, \alpha_{\text {rec }}$, and $f_{\text {ion }}=i / 8$ is that, although it is possible to ionize $\mathrm{O}$ I and $\mathrm{O}$ II within a few years for a characteristic value of $\epsilon_{\text {ion }} \sim 0.01, t_{\text {ion }}$ can easily become much longer than decades for higher ionization states. Note also the sensitive dependence on $t_{\text {ion }}$ with the magnetic field, such that for $B_{14}=3$ even O I will remain neutral for decades $\left(f_{\text {ion }} \approx 0\right)$ for $\epsilon_{\text {ion }}=0.01$. We thus conclude that on timescale of decades $f_{\text {ion }}$ will vary from $\approx 0$ to $\approx 2 / Z \sim 0.25$. 
Because it is very unlikely that $10 M_{\odot}$ of oxygen will be completely ionized on timescales of interest, $\mathrm{X}$-ray photons of energy $\sim 1-10 \mathrm{keV} \gtrsim h \nu_{\text {th }}=0.87 \mathrm{keV}$ will be strongly attenuated by photo-electric absorption. The X-ray optical depth is given by

$$
\tau_{X} \approx \frac{\rho_{\mathrm{ej}} \sigma_{\mathrm{bf}} R_{\mathrm{ej}}}{A m_{p}} \approx 100\left(\frac{E_{X}}{1 \mathrm{keV}}\right)^{-3} t_{10}^{-2} v_{9}^{-2},
$$

such that the ejecta will remain opaque to $\mathrm{X}$-rays of energies $E_{\mathrm{X}} \lesssim$ few $\mathrm{keV}$ for a decade or longer.

\section{References}

Atoyan, A. M. 1999, A\&A, 346, L49

Barbary, K., Dawson, K. S., Tokita, K., et al. 2009, ApJ, 690, 1358 Beloborodov, A. M. 2013, ApJ, 777, 114

Bietenholz, M. F., Frail, D. A., \& Hester, J. J. 2001, ApJ, 560, 254 Bietenholz, M. F., Kassim, N., Frail, D. A., et al. 1997, ApJ, 490, 291

Bucciantini, N., Quataert, E., Arons, J., Metzger, B. D., \& Thompson, T. A. 2008, MNRAS, 383, L25

Caleb, M., Flynn, C., Bailes, M., et al. 2016, MNRAS, 458, 718

Cano, Z., Johansson Andreas, K. G., \& Maeda, K. 2016, MNRAS, 457, 2761

Castro Cerón, J. M., Michałowski, M. J., Hjorth, J., et al. 2010, ApJ, 721, 1919

Champion, D. J., Petroff, E., Krarmer, M., et al. 2016, MNRAS, 460, L30

Chatterjee, S., Law, C. J., Wharton, R. S., et al. 2017, arXiv:1701.01098

Chatzopoulos, E., Wheeler, J. C., Vinko, J., Horvath, Z. L., \& Nagy, A. 2013, ApJ, 773, 76

Chen, K.-J., Woosley, S. E., \& Sukhbold, T. 2016, ApJ, 832, 73

Chevalier, R. A. 1998, ApJ, 499, 810

Chevalier, R. A., \& Li, Z.-Y. 1999, ApJL, 520, L29

Connor, L., Lin, H.-H., Masui, K., et al. 2016, MNRAS, 460, 1054

Cordes, J. M., \& Wasserman, I. 2016, MNRAS, 457, 232

DeLaunay, J. J., Fox, D. B., Murase, K., et al. 2016, ApJL, 832, L1

Deng, W., \& Zhang, B. 2014, ApJL, 783, L35

Drout, M. R., et al. 2013, ApJ, 774, 58

Drout, M. R., et al. 2014, ApJ, 794, 23

Duncan, R. C., \& Thompson, C. 1992, ApJL, 392, L9

Frail, D. A., Kulkarni, S. R., Sari, R., et al. 2001, ApJL, 562, L55

Fruchter, A. S., Levan, A. J., Strogler, L., et al. 2006, Natur, 441, 463

Gaensler, B. M., \& Slane, P. O. 2006, ARA\&A, 44, 17

Gal-Yam, A. 2012, Sci, 337, 927

Gao, H., Li, Z., \& Zhang, B. 2014, ApJ, 788, 189

Giannios, D. 2010, MNRAS, 403, L51

Greiner, J., Mazzali, P. A., Kann, D. A., et al. 2015, Natur, 523, 189

Hjorth, J., Sollerman, J., Moller, P., et al. 2003, Natur, 423, 847

Howell, D. A., Kasen, D., Lidman, C., et al. 2013, ApJ, 779, 98

Japelj, J., Vergani, S. D., Salvaterra, R., et al. 2016, A\&A, 590, A129

Johnston, S., Keane, E. F., Bhandari, S., et al. 2017, MNRAS, 465, 2143

Karastergiou, A., Chennamangalam, J., Armour, W., et al. 2015, MNRAS, 452,1254

Kasen, D., \& Bildsten, L. 2010, ApJ, 717, 245

Kasen, D., Metzger, B. D., \& Bildsten, L. 2016, ApJ, 821, 36

Kashiyama, K., \& Murase, K. 2017, arXiv

Katz, J. I. 2016, ApJ, 826, 226

Keane, E. F., Johnston, S., Bhandari, S., et al. 2016, Natur, 530, 453

Keane, E. F., Stappers, B. W., Kramer, M., \& Lyne, A. G. 2012, MNRAS, 425, L71

Kennel, C. F., \& Coroniti, F. V. 1984, ApJ, 283, 694

Kleiser, I. K. W., \& Kasen, D. 2014, MNRAS, 438, 318

Kouveliotou, C., Dieters, S., Strohmayer, T., et al. 1998, Natur, 393, 235

Kulkarni, S. R., Ofek, E. O., \& Neill, J. D. 2015, arXiv:1511.09137

Law, C. J., Bower, G. C., Burke-Spolaor, S., et al. 2015, ApJ, 807, 16

Levesque, E. M., Kewley, L. J., Berger, E., \& Zahid, H. J. 2010, AJ, 140, 1557

Liu, Y.-Q., \& Modjaz, M. 2016, arXiv:1612.07321

Lorimer, D. R., Bailes, M., McLaughlin, M. A., Narkevic, D. J., \& Crawford, F. 2007, Sci, 318, 777

Lunnan, R., Chornock, R., Berger, E., et al. 2014, ApJ, 787, 138
Lyubarsky, Y. 2014, MNRAS, 442, L9

Lyutikov, M. 2017, arXiv:1701.02003

Lyutikov, M., Burzawa, L., \& Popov, S. B. 2016, MNRAS, 462, 941

Maeda, K., Nakamura, T., Nomoto, K., et al. 2002, ApJ, 565, 405

Marcote, B., Paragi, Z., Hessels, J. W. T., et al. 2017, arXiv:1701.01099

Margalit, B., \& Piran, T. 2015, MNRAS, 452, 3419

Masui, K., Lin, H.-H., Sievers, J., et al. 2015, Natur, 528, 523

McCrum, M., Smartt, S. J., Rest, A., et al. 2015, MNRAS, 448, 1206

Medin, Z., \& Lai, D. 2010, MNRAS, 406, 1379

Mesler, R. A., Pihlström, Y. M., Taylor, G. B., \& Granot, J. 2012, ApJ, 759, 4

Metzger, B. D., Giannios, D., Thompson, T. A., Bucciantini, N., \& Quataert, E. 2011, MNRAS, 413, 2031

Metzger, B. D., Margalit, B., Kasen, D., \& Quataert, E. 2015, MNRAS, 454,3311

Metzger, B. D., \& Piro, A. L. 2014, MNRAS, 439, 3916

Metzger, B. D., Thompson, T. A., \& Quataert, E. 2007, ApJ, 659, 561

Metzger, B. D., Vurm, I., Hascoët, R., \& Beloborodov, A. M. 2014, MNRAS, 437, 703

Modjaz, M., Kewley, L., Kirshner, R. P., et al. 2008, AJ, 135, 1136

Mösta, P., Ott, C. D., Radice, D., et al. 2015, Natur, 528, 376

Murase, K., Kashiyama, K., \& Mészáros, P. 2016, MNRAS, 461, 1498

Nahar, S. N., \& Pradhan, A. K. 1997, ApJS, 111, 339

Nicholl, M., Berger, E., Smartt, S. J., et al. 2016, ApJ, 826, 39

Nicholl, M., Smartt, S. J., Jerkstand, A., et al. 2014, MNRAS, 444, 2096

Nicholl, M., Smartt, S. J., Jerkstand, A., et al. 2015, arXiv:1503.03310

Palmer, D. M., Barthelmy, S., Gehrels, N., et al. 2005, Natur, 434, 1107

Perley, D. A., Quimby, R. M., Yan, L., et al. 2016a, ApJ, 830, 13

Perley, D. A., Tanvir, N. R., Hjorth, J., et al. 2016b, ApJ, 817, 8

Petroff, E., Barr, E. D., Jameson, A., et al. 2016, PASA, 33, e045

Piro, A. L. 2016, ApJL, 824, L32

Popov, S. B., \& Postnov, K. A. 2013, arXiv:1307.4924

Popov, S. B., \& Pshirkov, M. S. 2016, MNRAS, 462, L16

Quimby, R. M., Aldering, G., Wheeler, J. C., et al. 2007, ApJL, 668, L99

Quimby, R. M., Kulkarni, S. R., Kasliwal, M. M., et al. 2011, Natur, 474, 487

Rane, A., Lorimer, D. R., Bates, S. D., et al. 2016, MNRAS, 455, 2207

Ravi, V., Shannon, R. M., \& Jameson, A. 2015, ApJL, 799, L5

Rhoads, J. E. 1997, ApJL, 487, L1

Rowlinson, A., Bell, M. E., Murphy, T., et al. 2016, MNRAS, 458, 3506

Scholz, P., Spitler, L. G., Hessels, J. W. T., et al. 2016, ApJ, 833, 177

Schulze, S., Kruhler, T., Leloudas, G., et al. 2016, arXiv:1612.05978

Sironi, L., \& Spitkovsky, A. 2011, ApJ, 741, 39

Sironi, L., \& Spitkovsky, A. 2014, ApJL, 783, L21

Spitkovsky, A. 2006, ApJL, 648, L51

Spitler, L. G., Cordes, J. M., Hessels, J. W. T., et al. 2014, ApJ, 790, 101

Spitler, L. G., Scholz, P., Hessels, J. W. T., et al. 2016, Natur, 531, 202

Stanek, K. Z., Gnedin, O. Y., Beacom, J. F., et al. 2006, AcA, 56, 333

Stanek, K. Z., Matheson, T., Garnavich, P. M., et al. 2003, ApJL, 591, L17

Suzuki, A., \& Maeda, K. 2016, arXiv:1612.03911

Tauris, T. M., Langer, N., \& Podsiadlowski, P. 2015, MNRAS, 451, 2123

Tendulkar, S. P., Kaspi, V. M., \& Patel, C. 2016, ApJ, 827, 59

Tendulkar, S. P., Bassa, C. G., Cordes, J. M., et al. 2017, arXiv:1701.01100

Thompson, C., \& Duncan, R. C. 1993, ApJ, 408, 194

Thompson, T. A., Chang, P., \& Quataert, E. 2004, ApJ, 611, 380

Thornton, D., Stappers, B., Bailes, M., et al. 2013, Sci, 341, 53

Usov, V. V. 1992, Natur, 357, 472

van Eerten, H., Zhang, W., \& MacFadyen, A. 2010, ApJ, 722, 235

Vergani, S. D., Salvaterra, R., Japelj, J., et al. 2015, A\&A, 581, A102

Verner, D. A., Ferland, G. J., Korista, K. T., \& Yakovlev, D. G. 1996, ApJ, 465,487

Wanderman, D., \& Piran, T. 2010, MNRAS, 406, 1944

Wheeler, J. C., Yi, I., Höflich, P., \& Wang, L. 2000, ApJ, 537, 810

Williams, P. K. G., \& Berger, E. 2016, ApJL, 821, L22

Woosley, S. E. 2010, ApJL, 719, L204

Wygoda, N., Waxman, E., \& Frail, D. A. 2011, ApJL, 738, L23

Yamasaki, S., Totani, T., \& Kawanaka, N. 2016, MNRAS, 460, 2875

Yang, Y.-P., Zhang, B., \& Dai, Z.-G. 2016, ApJL, 819, L12

Zhang, B. 2014, ApJL, 780, L21

Zhang, W., \& MacFadyen, A. 2009, ApJ, 698, 1261

Zrake, J., \& Arons, J. 2016, arXiv 